\title{
Philosophiques
}

\section{Ironie, valeurs cognitives et bêtise}

\section{Kevin Mulligan}

Volume 35, numéro 1, printemps 2008

Les valeurs de l'ironie (1) et le scepticisme à l'âge classique (2)

URI : https://id.erudit.org/iderudit/018237ar

DOI : https://doi.org/10.7202/018237ar

Aller au sommaire du numéro

Éditeur(s)

Société de philosophie du Québec

ISSN

0316-2923 (imprimé)

1492-1391 (numérique)

Découvrir la revue

Citer cet article

Mulligan, K. (2008). Ironie, valeurs cognitives et bêtise. Philosophiques, 35(1), 89-107. https://doi.org/10.7202/018237ar

\section{Résumé de l'article}

Cet article examine les théories de l'ironie comme feintise de Jancke (1929) et de Currie (2007) et défend une théorie de la feintise selon laquelle les évaluations de l'ironiste sont plus importantes que ses émotions, négatives et positives, et portent principalement sur les valeurs cognitives. L'objet formel de l'ironie est la bêtise et non pas une autre quelconque valeur négative éthique, politique ou esthétique. Je suggère que la conception de l'ironie ici esquissée ressemble à bien des égards à celle qu'on trouve dans les écrits de Robert Musil.
Ce document est protégé par la loi sur le droit d'auteur. L'utilisation des services d'Érudit (y compris la reproduction) est assujettie à sa politique d'utilisation que vous pouvez consulter en ligne.

https://apropos.erudit.org/fr/usagers/politique-dutilisation/ 


\title{
Ironie, valeurs cognitives et bêtise
}

\author{
KEVIN MULLIGAN \\ Université de Genève
}

\begin{abstract}
RÉSUMÉ. Cet article examine les théories de l'ironie comme feintise de Jancke (1929) et de Currie (2007) et défend une théorie de la feintise selon laquelle les évaluations de l'ironiste sont plus importantes que ses émotions, négatives et positives, et portent principalement sur les valeurs cognitives. L'objet formel de l'ironie est la bêtise et non pas une autre quelconque valeur négative éthique, politique ou esthétique. Je suggère que la conception de l'ironie ici esquissée ressemble à bien des égards à celle qu'on trouve dans les écrits de Robert Musil.
\end{abstract}

\begin{abstract}
This paper examines the pretence theories of irony of Jancke (1929) and Currie (2007) and argues for a pretence theory according to which the evaluations of the ironist are more important than his emotions, negative and positive, and bear principally on cognitive values. The formal object of irony is foolishness and not any ethical, political or aesthetic disvalue. I suggest that the view of irony outlined resembles in many respects the conception of irony to be found in the writings of Robert Musil.
\end{abstract}

\section{Introduction}

Pascal est assis dans un café près de la Sorbonne. Ses interlocuteurs babillent au sujet de Badiou et de l'abject, de l'ensemble vide et du phallus. Pascal se penche en avant. Avec toutes les apparences de sérieux, il dit: "Oui oui, on pourrait même employer ton assomption. » Pascal se tourne vers l'homme à sa gauche: "...et étendre ton argument ». Il s'adresse à l'homme à sa droite : "...l'ensemble vide serait alors identique au phallus. » Ses interlocuteurs font un signe de tête pour signifier leur accord fasciné. Mais ils ne sont pas tous stupides. L'un d'eux commence à se demander si Pascal est vraiment sérieux et, remarquant la trace d'un sourire presque béat sur son visage, décide qu'il ne l'est pas. Un autre hésite, oscillant entre les deux options. Un troisième est ravi de la contribution de Pascal et poursuit son idée : "En effet, et alors... ". Tagliatesta, qui suivait la conversation d'une table à côté, éclate de rire en voyant le sourire de Pascal. (J'ai simplifié ce récit d'une conversation parisienne; en particulier, j'ai supposé, de façon irréaliste, que les contributions à une telle conversation se produisent l'une après l'autre et que les participants s'écoutent les uns les autres).

La remarque de Pascal est ironique. Son état d'esprit est souvent ironique; et de fait, il est vraiment ironique. Qu'est-ce que cela veut dire que d'affirmer d'une remarque, d'une action ou d'un état d'esprit quelconque qu'il est ironique? Qu'est-ce que cela veut dire que d'affirmer d'une personne, réelle ou fictive, par exemple d'un narrateur, qu'il est ironique? Qu'est-ce que l'ironie, 
cette chose que les enfants, les femmes et les Américains sont incapables de comprendre ou d'apprécier, comme les Européens, méchants, âgistes et sexistes, le disaient dans les mauvais vieux jours?

Dans ce qui suit, je vais esquisser deux versions interreliées d'une réponse à certaines de ces questions: l'ironie est affaire de feintise $(\mathbb{2})$, et appliquer ces réponses à certains cas complexes d'ironie $(\mathbb{3} 3)$. Je vais enfin considérer deux questions $(\mathbb{S}$ ): 1) Qu'est-ce que la feintise et la feintise ironique ? 2) Quel est le but ou la fin de l'ironie ? - et je vais tenter de fournir une réponse partielle à la première question en donnant une réponse à la seconde.

\section{L'ironie en tant que feintise}

Les théories de la feintise affirment que feindre de F-er (d'asserter, de juger, de demander, de s'exclamer, d'approuver...) est essentiel au comportement ironique. Dans sa récente et remarquable description de l'ironie comme feintise, Currie cite certaines descriptions traditionnelles de l'ironie qui font appel au rôle de la feintise (Currie, 2006, p. 112 n. 3 ; cf. Jankélévitch, 1964, p. 68 ; Schaerer, 1941, 184). Bergson semble aussi avoir pensé que la feintise était essentielle à l'ironie :

Tantôt on énoncera ce qui devrait être en feignant de croire que c'est précisément ce qui est : en cela consiste l'ironie. Tantôt, au contraire on décrira minutieusement et méticuleusement ce qui est, en affectant de croire que c'est bien là ce que les choses devraient être: ainsi procède souvent l'humour (Bergson, 1959, 447).

Mais, pour autant que je sache, la première analyse de l'ironie en tant que feintise est celle donnée par Rudolf Jancke en 1929 dans Das Wesen der Ironie. Eine Strukturanalyse ihrer Erscheinungsformen (L'essence de l'ironie. Une analyse structurelle des différentes formes du phénomène). Jancke mentionne "des exemples d'ironie qui sont autant de gifles pour toutes les définitions éculées selon lesquelles l'ironie revient à dire le contraire de que l'on veut dire» (Jancke, 1929, 21).

Currie va plus loin:

Traditionnellement, on a pensé que l'ironie impliquait de dire une chose et de penser son contraire [...]. Pour une théorie qui a survécu si longtemps, elle explique incroyablement peu (Sperber \& Wilson 1981) (Currie 2006, p. 111-112).

Au cœur de la conception de Jancke se trouve l'idée que :

«Et Brutus est un homme honorable» est le pseudo-jugement (Scheinurteil) qu'avance l'ironiste (Jancke 1929, 22, cf. 10, 42).

Jancke ne croit pas cependant que la simulation ou la feintise (Simulieren, Fingieren: Jancke, 23, 41, 54, 66) de l'ironiste se limite aux pseudo-jugements. Par exemple, il y a des pseudo-questions (Scheinfrage, Jancke, 1929, 56) et des pseudo-exclamations ironiques. 
Deux objets, deux feintises

Jancke affirme que l'objet de l'ironie est toujours double : «[...] le regard de l'ironiste se dirige non seulement vers un objet [...] mais aussi vers son auteur. Si un artiste vous montre un de ses tableaux et vous demande votre opinion, le "très beau" que vous prononcez ironiquement est dirigé vers le tableau et vers l'artiste» (Jancke, 1929, 14, cf. Schaerer, 1941, 184) :

Il peut sembler à première vue qu'il n'y a pas deux objets, quand, par exemple, l'ironie est dirigée vers l'apparence, l'attitude, les actes ou le type de comportement d'une personne. Mais dans de tels cas le second objet est la personne elle-même [...] et le premier est son apparence, son attitude, ses actes ou le type de son comportement, comme modes d'apparence externes produits par la personne. L'ironie s'attaque toujours en dernière analyse au moi psychique d'une personne [...] elle voit la personne à travers les apparences (Jancke, 1929, 17).

[...] L'ironiste doit se mettre au diapason (sich einfüblen) de l'esprit de la personne qui sera l'objet de son ironie. Il ironise en empruntant pour ainsi dire l'opinion, l'intention, l'émotion, etc., de son adversaire et en les exprimant tout «comme si » elles étaient les siennes (Jancke, 1929, 12).

Jancke ne réussit pas à mettre complètement en évidence le fait que l'ironiste ne peut s'attaquer à l'objet de son ironie en feignant de faire siennes les opinions, les émotions et les intentions de sa victime que s'il feint également d'être un certain type de personne. Comme l'expose Currie:

La feintise qu'on entame avec de l'ironie est partiellement une feintise comportementale; on feint d'être en train de faire quelque chose qu'on ne fait pas: parler sérieusement et affirmativement, poser sérieusement une question, exprimer sérieusement son dégoût. Mais la feintise au fondement de l'ironie n'est pas une feintise de faire; c'est une feintise d'être. En faisant semblant d'affirmer ou en faisant semblant d'autre chose, on fait semblant d'être un certain type de personne - une personne ayant un point de vue restreint ou déficient sur le monde ou une partie du monde (Currie, 2007, 116).

L'ironiste et la victime ne sont pas les seules personnes essentielles à l'ironie :

Trois personnes au moins sont essentielles à l'ironie: l'ironiste, la personne qui est l'objet de l'ironie, et le spectateur idéal (Jancke, 1929, 46).

C'est dans sa description du spectateur idéal que Jancke se rapproche le plus de l'affirmation selon laquelle l'ironiste ne doit pas seulement feindre de F mais aussi feindre d'être une autre personne. L'ironiste est lui-même le spectateur idéal de la situation ironique. Le spectateur idéal "voit l'ironie comme s'il était quelqu'un d'autre ». C'est ce qui permet à l'ironiste d'apprécier l'ironie, peu importe si, oui ou non, personne d'autre n'est, ou ne semble être, conscient de l'ironie (Jancke, 1929, p. 46).

Le philosophe genevois René Schaerer remarque un des rôles de la feintise d'être dans l'ironie de Socrate: 
Socrate feint de prendre au sérieux l'éloquence de Gorgias, l'encyclopédisme d'Hippias, la piété d'Euthyphron. Il a devant lui des personnages admirés, il les suppose admirables. Il met ainsi l'individu en face d'un être fictif représentant ses propres prétentions prises au sérieux; il l'écrase sous sa propre image (Schaerer, 1941, 190; cf Longo 2007).

\section{Auto-ironie}

Parce que l'ironie implique toujours une trinité de personnes (Jancke, 1929, 78), Jancke affirme (de manière plutôt maladroite) que « dans sa structure essentielle, elle est tout à fait un phénomène social » (Jancke, 1929, 46). Il ne veut pas dire par là que l'ironie est essentiellement communicative. Il ne pense pas non plus que l'objet de l'ironie doive être distinct de l'ironiste. Une seule et même personne peut jouer les trois rôles de l'ironiste, de l'objet de l'ironie et du spectateur idéal. Car il y a de l' "allo-ironie" (Fremdironie) tout comme il y a de l'auto-ironie (Selbstironie). Dans «l'auto-ironie, la trinité des personnes est comprimée en une seule personne" (Jancke, 1929, p. 78). Jancke donne l'exemple suivant d'une ironie solitaire :

On peut par exemple trouver une image très belle et mettre soudainement un terme à nos panégyriques pour continuer ironiquement « oui, vraiment très beau » (Jancke, 1929, 78).

Currie en donne un exemple bien anglais :

Lorsque je sors sans mon parapluie et que je me heurte à une forte pluie, le «formidable! » que je prononce est un commentaire ironique sur l'insuccès qui m'est uniquement destiné (Currie, 2007, p. 115).

Une des erreurs impliquées ou suggérées par la description de l'ironie comme affirmant l'opposé de ce que l'on pense relève d'une description trop simpliste de ce que vise l'ironiste :

Jusqu'ici nous avons implicitement supposé qu'au jugement ironique «très beau » correspond nécessairement un «très mauvais » [...]. Cette supposition n'est pas nécessaire [...] dans les formes plus fines de l'ironie elle ne vaut pas du tout [...] (Jancke, 1929, 61).

Ce qui est «visé» (Gemeinte) ne doit pas toujours être l'opposé de ce qui est dit, mais doit se trouver dans la sphère négative ou positive opposée à celle de ce qui est dit (Vorzeichensphäre); pour l'ironiste lui-même ce qui est visé peut errer de façon très vague dans sa sphère sans être fixé à un point précis. Pour l'adversaire de l'ironiste le vague existe également pour peu qu'il relève l'ironie (Jancke, 1929, 62).

\section{Comme l'expose Currie:}

Ainsi, ce qui est important, c'est que l'énoncé de l'ironiste soit une manifestation du fait qu'il ou elle fait semblant d'avoir une position ou un point de vue restreint ou déficient, $\mathrm{F}$, et de la sorte, il nous met dans l'esprit d'une certaine perspective, d'un certain point de vue ou position, G (qui peut être identique à 
F ou simplement lui ressembler) étant la cible du commentaire ironique (Currie, 2007, 118).

\section{Exemples}

Deux illustrations importantes de la variété des feintises sont l'ironie feinte, ou pseudo-ironie, et ce que Currie appelle les « faire-semblant ironiques » (ironic pretendings) (Currie, 2006, 119).

\section{La pseudo-ironie}

Les énoncés et le comportement ironiques ont une dimension sérieuse et une dimension de faire-semblant, mais, pris comme tout, ils sont sérieux. C'est pourquoi il y a une possibilité de pseudo-ironie ou d'ironie feinte (Scheinironie, Jancke, 1929, 15, 63, 77). Jancke donne comme exemple la situation dans laquelle un artiste vous montre un de ses tableaux et vous demande votre opinion. Vous détestez l'artiste mais vous ne détestez pas le tableau. Votre remarque "très beau » est une instance de pseudo-ironie (Jancke, 1929, 15):

Le pseudo-ironiste se comporte "comme s'il » était ironique. Il n'a pas de vrais sentiments à l'encontre de ce qui semble être l'objet de son ironie, ses sentiments aussi sont simulés (fingiert) (Jancke, 1929, 63 ; italique ajouté).

Jancke donne deux exemples d'ironie feinte chez Proust :

Le docteur Cottard ne savait jamais d'une façon certaine de quel ton il devait répondre à quelqu'un, si son interlocuteur voulait rire ou était sérieux. Et à tout hasard il ajoutait à toutes ses expressions de physionomie l'offre d'un sourire conditionnel et provisoire dont la finesse expectante le disculperait du reproche de naïveté, si le propos qu'on lui avait tenu se trouvait avoir été facétieux. Mais comme pour faire face à l'hypothèse opposée il n'osait pas laisser ce sourire s'affirmer nettement sur son visage, on y voyait flotter perpétuellement une incertitude où se lisait la question qu'il n'osait pas poser [...] (Du côté de chez Swann, Bouquins, p. 176).

- Mais ce sera un bonheur..., commençait à répondre Swann, quand le docteur l'interrompit d'un air moqueur. En effet, ayant retenu que dans la conversation l'emphase, l'emploi de formes solennelles, était suranné, dès qu'il entendait un mot grave dit sérieusement comme venait de l'être le mot "bonheur ", il croyait que celui qui l'avait prononcé venait de se montrer prudhommesque. Et si, de plus, ce mot se trouvait figurer par hasard dans ce qu'il appelait un vieux cliché, si courant que ce mot fût d'ailleurs, le docteur supposait que la phrase commencée était ridicule et la terminait ironiquement par le lieu commun qu'il semblait accuser son interlocuteur d'avoir voulu placer, alors que celui-ci n'y avait jamais pensé.

Un bonheur pour la France ! s'écria-t-il malicieusement en levant les bras avec emphase (Du côté de chez Swann, Bouquins, p. 180 ; italique ajouté). 
La pseudo-ironie du docteur Cottard est une ruse et elle est délibérée. Mais l'exemple de pseudo-ironie donné plus haut par Jancke est, selon lui, un échec à être ironique (Jancke, 1929, 64).

Jancke croit qu'il y a une combinaison particulière d'ironie et de pseudoironie, une fusion qu'il décrit comme une «ironie de la pseudo-ironie». Supposons que je dise de votre tableau, sur un ton de voix ironique : «Je le trouve très réussi » et, que dans les faits, je sois convaincu que c'est un tableau très réussi. Je veux peut-être vous encourager à faire mieux. «Ici, la pseudo-ironie est ellemême voulue ironique." (Jancke, 1929, 64-65).

Est-il possible d'être ironique à propos de l'ironie? Y a-t-il une ironie de l'ironie ? Bien sûr, je peux être ironique à propos d'énoncés et d'états d'esprit ironiques passés. Toutefois, affirme Jancke en passant :

une ironie d'ironie, à la différence de l'ironie d'ironie simulée [...] est impossible au sein de l'unité d'un acte (Jancke, 1929, p. 65).

Si cela était juste, il serait erroné d'affirmer que Socrate, Érasme, Swift et Musil atteignent parfois des sommets consistant à ironiser leur propre ironie (sur Socrate, Érasme et Swift, cf. Kaiser 1963, p. 37-38). Leurs prouesses en ironie devraient plutôt être décrites comme des ironies sur la pseudo-ironie ou des ironies à propos de leurs assertions ou comportements ironiques. Au paragraphe 5 , nous évoquerons une autre manière de comprendre le fait d'ironiser sur l'ironie.

\section{Encore l'auto-ironie}

Les banals exemples donnés au premier paragraphe suffisent à montrer que l'auto-ironie est possible. Mais une description de l'auto-ironie en termes de feintise n'est aucunement simple. Jancke note deux types de cas différents: premièrement, je suis ironique à propos de mon «moi passé mort »; deuxièmement, je suis ironique à propos de mon « moi passé vivant, c'est-à-dire un moi passé et un moi possiblement futur du même type que mon moi passé ». Dans le premier cas, ma relation à l'objet de mon ironie ressemble à mon attitude envers une autre personne. Pour cette raison, Jancke penche pour le point de vue selon lequel il ne s'agit pas d'un type authentique d'auto-ironie:

On peut être ironique au sujet de ses propres actes et avis, au sujet des résultats de ses actes. La structure est la même que dans l'ironie dirigée vers autrui sauf que notre moi passé en est l'objet indirect; cela est tout à fait aisé pourvu qu'il y ait une distance, même infime, entre le moi présent et le moi passé (Jancke, 1929, 78).

On ne peut à la fois vouloir dire ou croire quelque chose intellectuellement et ironiser au sujet de cette opinion ou croyance ; cela voudrait dire que le moi pourrait simultanément dire « oui » et «non ». Le vouloir dire intellectuel doit toujours être objectifié avant que le moi ne s'élève à une ironie à son sujet et au sujet de son auteur, le moi antérieur (Jancke, 1929, 78). 
Une condition nécessaire pour l'auto-ironie authentique est une certaine continuité entre un soi affectif présent et passé (ou futur):

[...] la continuité du moi ne peut pas être complètement interrompue, il doit y avoir dans la conscience de soi un lien entre le moi passé et le moi présent (Jancke, 1929, 79).

La vraie auto-ironie se dirige seulement vers un moi passé vivant, vers un moi passé affectif. Si le sujet ironise sur un savoir faux [sic] au sujet d'un état de choses, il ironise sur son moi passé comme sur un autre soi (Jancke, 1929, 81).

Une autre condition nécessaire est la présence d'un substitut au spectateur idéal :

Le moi qui ironise sait qu'en ironisant sur le moi passé ou plus précisément sur les expériences de ce moi passé, il les frappe et les détruit [...]. Les sentiments véritables du moi passé deviennent maintenant des sentiments simulés du moi présent [...]. Simultanément les véritables sentiments du moi présent émergent et contrastent avec les sentiments simulés du moi passé. Mais puisqu'il y a un rapport de continuité vécue entre le moi présent et le moi passé [...] les sentiments simulés et véritables peuvent fusionner. Le moi présent subit ainsi une expérience fluctuante et «oscillante». Cela engendre l'état particulier de tension dans l'auto-ironiste qui ressemble à l'état de tension chez une personne qui est l'objet de l'ironie et ignore si l'ironiste veut vraiment dire ce qu'il dit ou pas ${ }^{1}$ [...] La possibilité d'une expérience oscillante constitue la possibilité de la véritable auto-ironie [...]. L'oscillation est donc un pendant de la dissimulation dans l'ironie dirigée vers autrui (Jancke, 1929, 80-81).

L'oscillation contribuerait ici à fonder le substitut au spectateur idéal (Jancke, 1929, p. 82).

Les ironies dramatiques, situationnelles et tragiques et les ironies du sort Currie définit l'ironie situationnelle en termes d'ironie dramatique:

Électra pleure sur les cendres dont elle pense qu'elles sont celles d'Oreste [...]. Par crédulité, pensée positive, ou simplement parce qu'ils ne connaissent pas les faits, les personnages de fiction ont souvent un point de vue limité, mis en relief par le point de vue plus inclusif qui est accordé à l'audience. Ainsi, l'ironie dramatique s'avère être simplement la représentation fictionnelle du type de contraste entre les points de vue dont on prétend être victime lorsqu'on parle ironiquement. L' «ironie situationnelle » est une catégorie gonflée qui menace d'engouffrer tout ce que nous trouvons surprenant, incongru ou injuste. Si on la restreint sensiblement, elle englobe les véritables circonstances qui reflètent celles de l'ironie dramatique, sauf qu'il est nécessaire que, sur le coup, il n'y ait pas de spectateur conscient de la disparité. C'était ironique [...] qu'elle démissionne un jour avant que sa promotion soit annoncée [...] (Currie 2006, p. 128129).

1. On voit ici que Jancke ne s'est pas complètement émancipé des emprises de la théorie traditionnelle de l'ironie. 
L' « ironie du sort » de Jancke tombe sous cette catégorie (gonflée) de l'ironie situationnelle:

[Dans] l'ironie du sort, le sort est conçu comme une personne impersonnelle [...]. Le sort fournit à l'homme tous les moyens nécessaires pour repousser un mal ou un autre inconvénient et arrange les choses de telle sorte que ce sont précisément ces moyens qui produisent un mal ou des inconvénients, ou de telle sorte que les moyens de protection ne sont pas touchés par le mal mais que ce qui devrait être protégé est détruit (Jancke, 1929, 75).

Un de ses exemples est celui de l'anthroposophe Rudolf Steiner qui, pendant la construction du Goethaneum, avait fait gainer tous les câbles électriques afin que les court-circuits ne puissent leur faire prendre feu. Or le Goethaneum a été détruit par un incendie, et seuls les câbles électriques y ont survécu.

Jancke croit que l'ironie tragique peut être définie en termes d'ironie du sort, les deux étant des exemples d'ironie de l'action en opposition à l'ironie des mots (Jancke 1929, p. 75). Il emploie la description du nœud tragique de Scheler :

Il est tragique au plus haut point que la même force qui permet à quelque chose de réaliser une valeur positive et élevée (dans la même chose ou dans une autre) devienne pendant son exercise la cause destructrice du porteur de cette valeur (Scheler, GW 3, 158, cf. tr. 183).

Si l'on suppose, derrière cette force active, un sort qui l'utilise pour nous duper $(f o p p t)$ «comme si » tout allait bien et engendre soudain le contraire, alors le phénomène de l'ironie tragique se dresse devant nous ; tragique car la force positive détruit, ironique car la force qui se présente comme positive se révèle être seulement apparemment positive parce qu'elle conduit en fait au mal (Jancke, 1929, 75-6; italique ajouté).

\section{La tête, le cœur, les valeurs, les points de vue et les feintes de l'ironiste}

Les analyses descriptives de l'ironie en termes de feintise doivent fournir des réponses à des questions telles que: Qu'est-ce que feindre? Qu'est-ce que feindre de F et feindre d'être une personne d'un certain type ? L'ironie implique-t-elle essentiellement deux différentes feintes?

Toute description de l'ironie devrait fournir des réponses à des questions telles que : pourquoi l'ironiste ironise-t-il ? Quels états d'esprit et attitudes, s'il y en a, sont essentiels à l'ironie ? Quelles sont les valeurs ou non-valeurs qui motivent l'ironiste?

Commençons par une remarque historique. La théorie de Jancke est construite sur des descriptions d'états mentaux, d'actes et d'activités " comme si », développées au début du vingtième siècle par des élèves de Brentano tels que Husserl et Meinong ainsi que par leurs élèves, tel que Roman Ingarden ( $c f$. Mulligan, 1999). Jancke emploie une version de ces descriptions, donnée par Alexander Pfänder, et s'y réfère. Selon les théories autrichiennes du fairesemblant (make-believe), des états et des actes «sérieux» tels que voir, 
entendre, juger, sentir et désirer ont tous des contreparties imaginatives : voir-comme-si, entendre-comme-si, juger-comme-si (supposer), sentir-comme-si, désirer-comme-si. Le voir-comme-si ressemble au voir, mais ce n'est pas une sorte de voir et il est sujet à la volonté. Ce qui est vrai du couple voir/voir-comme-si est vrai des autres couples. Une telle description est une description de certains actes, états et activités mentaux, ce n'est pas une description d'actes, d'activités ou d'actions publiques, qu'ils soient linguistiques ou non. Parmi les héritiers de Brentano, la distinction entre les actions publiques sérieuses et non sérieuses n'a cependant pas toujours été observée. Jancke en est justement un exemple. Il fait référence, comme nous l'avons vu, à des émotions simulées, des émotionscomme-si et des sentiments-comme-si mais aussi à des questions et des jugements (assertions) simulés. Or il n'est pas du tout évident que la propriété d'être comme-si d'une émotion-comme-si soit identique à la propriété d'être comme-si d'une question-comme-si. Meinong (1997, p. 383) emploie à la fois "émotion imaginative " et "pseudo-émotion " pour faire référence au même phénomène (tout comme "désir imaginatif » et "pseudo-désir »). Mais il n'est pas évident que la relation entre ce que Jancke appelle une "pseudo-question » et une question est celle qui, selon Meinong, a lieu entre une émotion feinte et une émotion.

Alors que les héritiers de Brentano sont partis d'une théorie des étatsmentaux-comme-si et des actes-mentaux-comme-si et qu'ils ont ensuite procédé à la description des assertions-comme-si, des promesses-comme-si et des questions-comme-si, la philosophie analytique est partie de descriptions des actions-comme-si et des actions feintes - Frege sur la présence ou l'absence de la force assertorique, Ryle, Austin, Searle et Anscombe sur la feintise et ensuite seulement, elle a proposé des descriptions d'émotions feintes, par exemple (Walton). Tout comme certains des héritiers de Brentano, la vaste littérature contemporaine sur la feintise, le faire-semblant et la simulation n’observe pas toujours la distinction entre les états-mentaux-comme-si et les actions-publiques-comme-si.

Qu'est-ce donc alors, pour l'ironiste, que de feindre de F-er ? On peut distinguer deux conceptions. La première est que lorsque l'ironiste feint d'affirmer, de poser une question, etc., il feint de croire ou de savoir quelque chose, il feint de désirer savoir quelque chose. Cette conception peut être combinée avec le point de vue selon lequel les assertions indiquent ou manifestent une croyance ou une connaissance, que les questions indiquent ou manifestent des désirs, etc. La considération qui est peut-être la plus importante en faveur de cette conception est que, dans beaucoup de cas d'ironie, l'ironiste essaie vraiment de dissimuler quelque chose ou de contrôler la divulgation de ses états d'esprit. D'autre part, l'ironie peut être complètement solitaire et non communicative. Dans de tels cas, on peut penser qu'il est plutôt absurde de dire que l'ironiste feint par rapport à lui-même d'être dans un certain état mental. 
Selon la seconde conception, si un ironiste feint d'affirmer ou de se renseigner, il n'est pas du même coup en train d'affirmer ou de se renseigner. Une version de ce point de vue assimile les actes de l'ironiste au comportement de l'acteur. Tout comme l'acteur peut énoncer une phrase assertorique et pourtant ne rien affirmer du tout, de la même façon, l'ironiste énonce une phrase assertorique mais n'affirme pas. Cependant une différence persiste entre l'acteur et l'ironiste. L'acteur ne feint pas de savoir ou de croire quoi que ce soit (bien qu'il puisse très bien feindre être une personne qui sait ou croit différentes choses). L'ironiste, à l'inverse, feint souvent qu'il croit, qu'il sait ou qu'il désire.

Jancke semble accepter les deux conceptions, car il accepte une thèse approuvée par d'autres héritiers de Brentano selon laquelle les promesses, les assertions et les questions non sincères ne sont pas des promesses, des assertions ou des questions. Mais cette thèse est très peu vraisemblable. Une promesse non sincère, la promesse de F-er en l'absence de toute intention de F-er, demeure une promesse ${ }^{2}$.

Feindre de F-er, comme Currie le remarque, n'est pas la seule feintise essentielle à l'ironie. L'ironiste feint d'être une personne d'un certain type ayant un certain point de vue. Un argument en faveur de cette affirmation se base sur le contraste entre l'ironie socratique, ou ironie pédagogique, et l'ironie au sens propre.

Dans l'ironie pédagogique, l'ironiste feint d'approuver une certaine proposition. De manière similaire, dans une preuve indirecte, on suppose qu'une certaine proposition est vraie. Une des différences entre les deux cas est que l'audience d'une preuve indirecte sait habituellement ce qui se passe. Pourquoi l'ironiste socratique ne donne-t-il pas simplement une preuve indirecte de la conclusion souhaitée? Parce qu'il veut stimuler l'objet de son ironie à une activité cognitive indépendante (Bollnow, 1947, 148-149). L'ironie pédagogique est l'exception principale à la règle selon laquelle l'ironie attire l'attention sur les points de vue d'une personne. L'objet de l'ironie pédagogique est simplement une croyance unique d'une personne:

[L]'ironie pédagogique porte sur une opinion particulière d'une personne, mais prend sérieusement cette personne comme un tout ; ['ironie non pédagogique] porte sur un homme compris comme un tout (Bollnow, 1947, 151. Cf. Jancke, 1929, p. 57-61).

L'ironie au sens propre n'est pas pédagogique. Elle implique de feindre d'être une personne d'un certain type ayant un certain point de vue. Je crois que la meilleure façon de le montrer est de partir d'une description des motifs de l'ironiste.

2. Il me semble que les objections formulées par White (White, 1990) et avant White par Collingwood à l'égard des emplois philosophiques de la feintise, sans ou avec l'intention de tromper, n'ont pas encore été digérées par la philosophie contemporaine de la feintise (pour un début, cf. Mulligan, 1999). 
Pourquoi l'ironiste feint-il ? Quelles attitudes dissimule sa feintise? Quelles attitudes veut-il exprimer, s'il veut exprimer quelque chose? On peut faire plusieurs choses en feignant, et l'ironiste, lui aussi, peut faire différentes choses en feignant. Il peut se moquer de sa cible, mépriser, être sarcastique, railler, dérider, persifler, ridiculiser, exposer, démasquer, désapprouver son souffre-douleur, sa victime. L'ironiste est souvent irrévérencieux, agressif. Il y a donc deux questions naturelles : l'ironie est-elle toujours motivée ainsi ? À quelle profondeur les motivations de l'ironiste doivent-elles être ancrées ? Les énoncés ironiques, on l'affirme souvent, expriment toujours ou sont toujours motivés par l'affront, le mépris, la désapprobation, la dérision, etc. :

[...] l'attitude exprimée par un énoncé ironique en est invariablement une du type du rejet ou de la désapprobation (Sperber \& Wilson, 1986, p. 239).

Currie objecte à juste titre :

[...] Lorsque je dis de ma fille : «elle est très excitée; le père Noël viendra cette nuit ", je feins, au service de l'ironie, d'affirmer que le père Noël viendra cette nuit. Mais je n'exprime pas d'hostilité, d'indignation, de mépris, de désapprobation ou de raillerie à l'égard de ma fille parce qu'elle y croit (Currie, 2007, p. 116).

Jancke cite une remarque analogue de Paulhan:

Il y a une ironie ailée et subtile. Il y a une ironie méchante, et une ironie dédaigneuse ou bienveillante, une ironie naïve, désabusée, du misanthrope, du philanthrope, de l'assassin, qui raille sa victime (Paulhan, 1914, 146-7).

Et il ajoute que bien que «le mépris [...] rende l'ironie "tranchante", l'amour peut rendre l'ironie gentille ou bienveillante, triste ou mélancolique, ludique» (Jancke, 1929, p. 74).

Nietzsche et d'autres ont souvent affirmé que l'ironie peut être motivée par l'attitude de respect de soi ou d'auto-révérence, que «l'ironie n'est qu'un des visages de la pudeur » (Jankélévitch, 1964, p. 165). On a dit de l'ironie de Renan qu'elle était le masque de la pudeur (Dugas, 1903, p. 483). Jancke écrit :

Dans sa fonction plus noble [l'ironie] n'est pas là pour railler (verspotten). Sa fonction consiste aussi à préserver les expériences les plus sacrées du moi le plus intime. L'ironie est ainsi le gardien du saint des saints, qui laisse entrer seul celui qui en est digne [...] (Jancke, 1929, 11, cf. Höffding, 1930, 69).

Bollnow décrit «l'ironie révérencieuse plutôt rare ", dont l'objet peut être soi-même ou un autre, de la manière suivante:

Là où la révérence ne peut suivre sa première inclination, à savoir d'être silencieuse, et que malgré ses inhibitions, un homme est forcé de parler, il se produit souvent, dans le conflit entre la nécessité de parler et la timidité enracinée, cette forme de discours indirect qu'on appelle l'ironie. Ici, l'ironie [...] est une expression de révérence [...]. Non seulement elle court le risque d'être mal comprise 
par les autres, mais elle peut trop facilement tourner en un comportement irrévérencieux (Bollnow, 1947, p. 147, cf. p. 59, 154).

[C]elui qui, par révérence, est privé de la possibilité d'exprimer immédiatement ses sentiments a l'impression que ce qui est saint ne peut être traité avec la familiarité grossière des formulations quotidiennes [...]. [L]a seule manière pour lui de s'en sortir est d'employer une formulation ironique de façon à confiner ce qu'il a dit à la manière dont il parle [...], de façon à indiquer quelque chose qui va au-delà du domaine du dicible (Bollnow, 1947, p. 174-5).

Selon le jugement le plus ancien et le plus commun sur la honte (pudeur, shame, pudor, aidôs) et la révérence, elles protègent et sont des "gardiens » (Hume, Kant, Joubert). Si c'est le cas, il n'est pas surprenant qu'elles profitent de l'ironie.

Si l'ironie peut être motivée par des attitudes aussi différentes, alors il est erroné d'affirmer qu'un type particulier d'attitude est essentiel à l'ironie. Effectivement, il est erroné d'affirmer que l'ironie requiert une attitude affective de quelque sorte que ce soit. Comme Jancke l'expose:

[...] il peut y avoir une ironie malveillante, naïve, amicale, hostile, etc. En d'autres termes, la coloration de l'ironie, que l'on exprime normalement avec l'aide d'un adjectif, ne dit rien du phénomène ironique lui-même mais décrit plutôt l'état passager de l'ironiste. Le phénomène ironique nu a une structure entièrement plate, dépourvue de couleur, sans aucune profondeur ou poids significatif (Jancke, 1929, 74).

Qu'est-ce donc qui meut l'ironiste ? Selon Grice :

[...] l'ironie est intimement liée à l'expression d'un sentiment, d'une attitude ou d'une évaluation (Grice, 1989, p. 53, italique ajouté).

Ici, " évaluation » pourrait référer à une croyance évaluative ou à un jugement évaluatif mais aussi à une certaine sensibilité ou à une conscience de la valeur. Y a-t-il des valeurs, positives ou négatives, qui motivent l'ironiste? Lesquelles?

Plusieurs remarques faites par Jancke et Currie pointent vers ce que je considère être la bonne direction :

[L']ironie appartient complètement à la sphère logique (Jancke, 1929, p. 47; mais cf. p. 17).

Dans un passage que j'ai déjà cité, Currie affirme que l'ironiste « feint d'être un certain type de personne - une personne ayant un point de vue restreint ou déficient du monde, ou d'une partie de celui-ci » (Currie, 2007, p. 116 ; italique ajouté). Il dit également :

Je crois que l'ironie est limitée par l'obligation de viser seulement des points de vue d'un certain type: ceux auxquels nous pouvons appliquer un standard de pertinence (reasonableness) [...] Les tendances à croire ou à désirer de certaines manières, ou à avoir certaines réactions émotionnelles, sont une proie rêvée, même 
dans les cas où aucun blâme personnel n'est attaché à la personne dont le point de vue a été isolé (Currie, 2007, p. 118 ; italique ajouté).

L'ironiste feint un point de vue limité : il feint de dire ou faire quelque chose que seulement quelqu'un qui n'a pas réussi à voir certains faits ou valeurs de manière bienveillante et vivante dirait ou ferait (Currie, 2007, p. 123, n. 23; italique ajouté).

Ce qui rend la remarque [«le père Noël viendra cette nuit »] ironique [...], c'est l'invitation, dans mon assertion feinte, à assister au point de vue épistémiquement limité [de la fille de Currie] (Currie, 2007, p. 116 ; italique ajouté).

C'est une chose de juger que quelqu'un est un mauvais peintre, qu'il est mesquin, injuste ou cruel. Mais c'est une chose complètement différente de juger que les raisons d'agir, de sentir, de désirer d'une personne, ses points de vue, sont plus ou moins inappropriés ou mauvais, ou qu'elle est victime d'auto-duperie. De manière similaire, les critiques ou la désapprobation basée sur des jugements de la première sorte diffèrent des critiques ou de la désapprobation basée sur des jugements de la deuxième sorte. Les points de vue qui sont plus ou moins déraisonnables ou limités épistémiquement sont certes de mauvaises choses. Ils appartiennent à la même famille de mauvaises choses à laquelle appartiennent la duperie de soi-même, l'erreur, l'illusion, l'ignorance, l'inexactitude et l'hypocrisie. Elles ont une valeur négative. Mais les valeurs pertinentes sont cognitives ou épistémiques. Et certaines d'entre elles sont également des vices cognitifs.

La structure de la famille des valeurs (positives et négatives) cognitives et des vices et vertus épistémiques est une affaire controversée. J'ai plaidé ailleurs en faveur de deux thèses (Mulligan, 2000). D'abord, la valeur cognitive centrale est la valeur de la connaissance. Les valeurs de vérité, de justification, de clarté, de précision, de justesse, d'intégrité intellectuelle, etc., dérivent de la valeur de la connaissance. Deuxièmement, l'aveuglement, l'indifférence et l'hostilité face aux valeurs cognitives constitue le vice cognitif par excellence : la bêtise (foolishness). Ainsi, la bêtise n'est pas la stupidité (cf. l'histoire de Pascal). Une conséquence immédiate de la deuxième thèse est que le postmodernisme est bête. Dans ce qui suit, je vais présumer que ces deux thèses sont correctes.

L'ironiste, comme je le suggère, est sensible à la bêtise et aux valeurs cognitives négatives. Ce qui pousse l'ironiste à ironiser, c'est son impression de bêtise et de valeurs cognitives négatives. Dans les exemples d'ironie auxquels les théoriciens de l'ironie se réfèrent le plus souvent, l'ironiste a aussi une attitude affective négative face à l'ironie. Mais cela ne doit pas être nécessairement le cas, comme nous l'avons vu. L'ironiste peut aussi être simplement amusé (cf. l'histoire de Pascal).

Un avantage du point de vue selon lequel l'objet formel de l'ironie est la bêtise est qu'il nous fournit immédiatement une façon plausible de distinguer entre l'ironie et la satire. Le satiriste est conscient de l'exemplification de valeurs 
éthiques et politiques négatives, et condamne ce qui exemplifie ces valeurs. C'est une affirmation habituelle et plausible. Mais si nous caractérisons le satiriste par les valeurs qui le meuvent, nous devrions aussi être en mesure d'identifier les valeurs qui motivent l'ironiste. L'ironiste est sensible aux illusions, aux erreurs, à la fausseté, à l'irrationalité, à l'ignorance, à l'inexactitude, au manque de rigueur, à la duperie de soi et à la folie, et s'en préoccupe souvent. La cible de l'ironie peut être cruelle, méchante, injuste ou politiquement mauvaise. Mais ce qui frappe l'ironiste, c'est le fait que sa cible a tort d'approuver le point de vue qu'elle adopte, que ses raisons sont mauvaises ou bien encore qu'elle est irrationnelle. Le point de vue de l'ironiste peut être celui de quelqu'un de cruel, méchant, injuste, corrompu ou politiquement mauvais, mais il feint de l'adopter de façon à attirer l'attention sur le fait qu'il est incorrect, irrationnel inapproprié ou bête.

Une raison de penser que l'objet formel de l'ironie est la bêtise ou quelque chose de ce genre est que cette conception explique une caractéristique bien connue de l'ironie. Le satiriste, comme tout critique, est motivé par des buts et des fins pratiques. Il veut changer le monde. Cela n'est pas vrai de l'ironiste en tant qu'ironiste. Même l'ironie qui vise à être comprise veut être comprise lentement, plutôt tard que tôt, et affiche ainsi le caractère quiétiste de l'ironie. "L'ironie, affirme Bollnow (1947, p. 159), est à l'extérieur de la sphère de l'utilité pratique.» Plus généralement, comme l'explique Jancke, l'ironiste est caractérisé par un type de « liberté intérieure » : il n'est pas " lié » par des considérations pratiques à l'objet de son ironie, il est au-dessus de la situation (Jancke, 1929, 20; 26-28; Schaerer, 1941, 185). Sous cet angle, l'ironie diffère du mensonge, de la raillerie, de la moquerie, du sarcasme et de la satire (Jancke, 1929, p. 28-29). Cet aspect de l'ironie s'explique aisément. Les valeurs positives et négatives qui font battre le cœur sont sensorielles (le plaisant et le déplaisant), éthiques (le mal, la générosité), vitales (santé, bien-être), politiques (liberté, injustice) ou esthétiques. Les valeurs cognitives par contre nous excitent moins que n'importe quel autre type de valeurs. Si le cœur de l'ironiste bat, il bat pour les valeurs cognitives - mais seulement faiblement. Comme le dit Rilke, l'ironie ne va jamais au fond des choses. Il n'y a qu'un contexte dans lequel les valeurs cognitives ont tendance à provoquer des sentiments forts. La bêtise, mais aussi la stupidité provoquent la condamnation, le mépris et d'autres sentiments forts lorsqu'ils sont des obstacles à la réalisation de valeurs non cognitives. Dans de tels cas, la valeur négative de la bêtise et de la stupidité est extrinsèque. Tout au plus, c'est faiblement que bat le cœur de l'ironiste. Mais il bat à cause de la valeur négative et intrinsèque de la bêtise.

L'ironiste ne veut influencer, convaincre, persuader ou réformer qui que ce soit, pas même dans les cas d'ironie communicative, où il cherche à ce que son ironie devienne visible, bien que graduellement. Comme le dit Jankélévitch (une telle) ironie "ne cherche pas à être crue, elle veut être comprise» (Jankélévitch, 1964, p. 60). Dans sa discussion de cas semblables, Jancke affirme que : 
L'ironiste courtise les « autres » en sa faveur. Mais sa sollicitation doit être caractérisée par une certaine indifférence, autrement l'ironie échoue et devient sarcasme ou mépris (Jancke, 1929, 48).

Une intense "activité de persuasion est immanente à l'ironie, qui n'en demeure pas moins indifférente à l'échec» (Jancke, 1929, p. 49). Bien sûr, l'ironie peut être inappropriée de différentes manières. "L'ironie ne peut jamais être fausse ou correcte, mais elle est stupide, ennuyeuse ou vulgaire » (Jancke, 1929, p. 17), ou encore, pourrions-nous ajouter, bête.

Supposons qu'il puisse y avoir quelque chose de tel qu'un mode grammatical de l'ironie, se manifestant par une pointe d'ironie ou quelque "smiley » grotesque, jaune ou mauve. À quel mode cela ressemblerait-il le plus? Il y a plusieurs modes qui sont liés à l'action, aux souhaits et à la volonté : le mode impératif, le mode précatif, le mode optatif, le mode hortatif, le mode consultatif, le mode invitatif, le mode performatif et même le mode vocatif. Mais certains modes ne sont pas vraiment liés à des actions, au souhait, à la volonté ou aux buts : le mode concessif, le mode exclamatif et le mode de l'expression du regret. Un mode de l'ironie serait à classer dans ce deuxième groupe dans la mesure où l'ironie est quiétiste.

Si la description précédente des motifs de l'ironiste et des valeurs qui le meuvent est correcte, alors cela explique pourquoi l'ironie requiert de l'ironiste qu'il feigne d'être une personne d'un certain type ayant un certain point de vue et feignant de F-er (où « F » porte sur des actions, linguistiques et non linguistiques, conventionnelles et non conventionnelles).

L'ironiste identifie la relation entre un type de personne, un type de point de vue et un type de comportement : compte tenu de son point de vue, il est plutôt irrationnel de F-er pour cette personne. Dans les cas les plus simples, la personne que l'ironiste feint d'être est sa cible, sa victime ou une personne du même genre, le point de vue est celui de la cible et l'action est une action de la cible ou d'une personne semblable à la cible. Un ami vous montre son dernier tableau. Vous feignez d'en faire l'éloge en vous exclamant ironiquement «qu'il est beau! ». Vous pensez que votre ami en ferait l'éloge de cette façon. Après tout, pourquoi vous le montre-t-il ? (cf. Jancke, 1929, p. 12). Vous feignez d'être une personne qui ferait l'éloge du tableau. Vous manifestez que l'éloge serait injustifiée, que votre ami est bête ou proche de l'être ou coupable de bêtise. Vous n'êtes pas en train de critiquer les carences esthétiques du tableau, même si l'ironie dépend de l'impression selon laquelle il n'est pas beau. Pascal feint de tirer une conclusion dont il pense que ses interlocuteurs la tireraient ou l'accepteraient, étant donné leurs points de vue d'intellectuels sorbonnards. Il feint d'être l'un d'eux. Il identifie précisément l'absurdité de tirer une telle conclusion, l'absurdité d'être le type de personne qui pourrait tirer une telle conclusion. La bêtise et l'irrationalité sont au fond des propriétés d'une personne, des propriétés qu'une personne a en vertu de sa relation à ses actions, ses pensées, ses sentiments et ses attitudes. C'est parce que l'ironiste 
est mu par la bêtise des gens qu'il doit feindre d'être une personne qui est bête et non seulement feindre de se comporter de façon bête.

\section{Ironies - petites, grandes, gonflées et autrichiennes}

La description précédente de l'ironie comme étant essentiellement une réaction à une folie (apparente) n'est-elle pas une schématisation, peut-être une schématisation absurde (et ainsi stupide, sinon même bête) ? Elle a au moins le mérite de capturer certaines des caractéristiques principales de l'ironie du plus grand ironiste du vingtième siècle, Robert Musil. Et cela pourrait très bien être le cas qu'une description réussie des meilleures instanciations d'espèces non naturelles telles que l'ironie est la meilleure description à donner de l'espèce.

La passion derrière L'Homme sans qualités, le chef d'œuvre de Musil, est une passion cognitive, nous dit l'auteur, «la passion pour la justesse/exactitude " (Richtigkeit/Genauigkeit, Musil, 1983, p. 1937). Son panorama ironique de la variété infinie de la bêtise (et par exemple de la philosophie proto-continentale) du début du vingtième siècle emploie l'ironie afin d'afficher et d'identifier précisément cette variété. Sa croisade ironique est soutenue par le fait que les variétés principales de la bêtise sur lesquelles il porte son regard sont toutes, pense-t-il, le résultat de fausses conceptions de la relation entre la Vie et l'Esprit et sont exemplifiées par des formes de vie enracinées dans de telles conceptions erronées. Les exemples dans son bêtisier sont tous cognitivement vicieux - aveugles, indifférents ou hostiles aux valeurs cognitives. Son héros, Ulrich, est un héros épistémique.

Mais la description précédente ne convient seulement qu'à une partie des ironies de Musil, et l'omission est typique de plusieurs philosophies de l'ironie. La description précédente est une description de ce qu'on a appelé la «petite ironie » en opposition à la "grande ironie » (Höffding, 1930, p. 67; Jancke, 1929, p. 2 ; $88 ; c f$. Bollnow, p. 156-257). Les petites ironies sont des épisodes ironiques, c'est-à-dire un comportement, un acte linguistique ou non linguistique. La "grande ironie » est employée en référence à deux choses apparemment différentes. D'abord, à l'ironie situationnelle, l'ironie des choses $^{3}$, l'ironie du sort ou l'ironie tragique $(c f . \$ 3)$ comprise au sens le plus gonflé qui soit. Ensuite, elle fait référence à certains états ou attitudes persistants. Les amis de la distinction entre la petite et la grande ironie plaident souvent largement en faveur de la dernière. Ainsi, Bollnow décrit une attitude ironique persistante envers soi-même (ou un autre) de la manière suivante:

On ne prend pas sa propre existence, dans son entier, au sérieux, ou plus exactement : la suspension (Schwebe, flottement) entre prendre au sérieux et ne pas prendre au sérieux, qui caractérise l'ironie, traverse notre propre vie comme un tout et crée une situation flottante particulière (Schwebesituation), à travers laquelle la relation à notre propre existence n'est pas rompue mais assouplie (Bollnow, 1947, p. 156, cf. p. 163).

3. Pour un catalogue remarquable de l'ironie des choses, voir Hofmannstahl, 1955. 
Et il affirme ensuite que :

Nous avons là, dans cette attitude ironique face à la vie, le véritable phénomène originaire de l'ironie [...] qui rend intelligibles tous les exemples précédents (Bollnow 1947, p. 157; italique ajouté).

Toute analyse de l'ironie devrait clarifier la relation entre les deux types de grande ironie et la relation entre la grande et la petite ironie.

On peut dire à la fois des remarques et des situations qu'elles sont " ironiques ", mais aussi qu'elles sont " comiques » et "drôles ». Dans le cas des états d'esprit, persistants ou éphémères, des remarques et d'autres activités, ainsi que des situations, on peut dire qu'ils sont « ironiques ", mais on ne peut dire qu'ils sont «comiques » ou «drôles » sans pour cela changer la signification des termes. L'ironie des situations et des choses a-t-elle quelque chose en commun avec l'ironie des remarques, du comportement et des états d'esprit?

Cette question ressemble à une autre question très discutée. On peut dire d'un paysage réel et d'un paysage fictif qu'ils sont beaux, d'une mélodie entendue et d'une mélodie imaginée qu'elles sont laides, d'un geste réel et d'un geste imaginé qu'ils sont élégants. Est-ce que la beauté ou la laideur des facta a quelque chose à voir avec la beauté ou la laideur des ficta? Une bonne réponse à cette question va comme suit : la beauté (la laideur, l'élégance) des facta est une propriété que les facta auraient même s'ils étaient des ficta. Les propriétés esthétiques d'une mélodie demeurent invariantes dans l'écoute et dans l'imagination de la mélodie. Ma suggestion est que, de manière similaire, l'ironie des choses et des situations est une propriété que les facta ont seulement si - et parce que - les ficta correspondants sont ironiques. Dans la mesure où les ficta dépendent de la feintise, toute ironie dépend de la feintise.

Les ironies de Musil sont grandes et petites. Les petites ironies sont à trouver partout dans les conversations de Ulrich, par exemple dans ses conversations avec le très intelligent - et très bête - Arnheim, ainsi qu'avec la bête et stupide Diotima. Les grandes ironies sont cependant déployées dans les relations entre le narrateur de L'Homme sans qualités, Ulrich, et leurs points de vue. Ce sont les grandes ironies en particulier qui illustrent ce que Musil, employant un adjectif de Kierkegaard, appelle «la vie flottante» (das schwebende Leben). Musil décrit cet idéal ironique de la manière suivante:

L'ironie, c'est représenter un clérical de telle sorte qu'un bolcheviste soit aussi atteint. Représenter un idiot de telle sorte que l'auteur sente soudainement : ça, c'est en partie moi. Cette espèce d'ironie, l'ironie constructive, est assez inconnue dans l'Allemagne d'aujourd'hui. Elle sort toute nue de la connexion entre les choses. On prend l'ironie pour raillement et moquerie (Musil, 1983a, 1939).

L'ironie qui émerge de la connexion entre les choses, comme Musil les dépeint, n'est pas socratique:

[Ironie] socratique. Feindre de ne pas savoir.

[Ironie] moderne. Ne pas savoir (Musil, 1983b, 920). 
Ainsi, Musil est intéressé par le cas limite dans lequel la supériorité cognitive de l'ironiste, le petit ironiste ou l'auteur, s'avère être simplement apparente ou possiblement apparente. En d'autres termes, le cas où la bêtise des personnes et des points de vue ironisés s'avère être un type de bêtise dont l'ironiste est un exemple, ou là où cela demeure une possibilité. L'ironie de ce genre est peutêtre le plus grand hommage qui puisse être adressé aux valeurs cognitives. Il est la source des principales valeurs esthétiques du roman de Musil, le comique sublime et le sublimement comique. C'est parce que c'est là son idéal ironique que Musil peut écrire : «Pour moi, l'ironie n'est pas un geste de supériorité mais une forme de combat » (Musil, 1983b, 941), et peut insister sur l'importance d' " une certaine sympathie dans toute ironie» (Musil, 1983c, 1148). Même les idiots de Musil sont parfois (involontairement) plus près de la vérité ou des vrais problèmes que son héros épistémique Ulrich, le protopositiviste logique. Mais la motivation de Musil n'est pas celle d'Érasme. C'est plutôt [qu']à la littérature appartient de manière essentielle ce qu'on ne sait pas; la révérence face à ce qu'on ne sait pas ${ }^{4}$ (Musil, 1983b, 972).

\section{Bibliographie}

Bergson, H. «Le Rire », in Euvres, Paris, PUF, 1959.

Bollnow, O. F. Die Ehrfurcht, Frankfurt, Klostermann, 1947.

Currie, G. "Why Irony is Pretence ", in S. Nichols, The Architecture of the Imagination.

New Essays on Pretence, Possibility, and Fiction, Clarendon Press, Oxford, 2006, 111-136.

Dugas, L. "La Pudeur », Revue philosophique de la France et de l'étranger, 1903, 468487.

Höffding, H. Humor als Lebensgefühl (Der grosse Humor) , Leipzig, O. R. Reisland, 1930.

Hofmannsthal, H. von. « Die Ironie der Dinge », Prosa IV, Gesammelte Werke, 1955, 40-44.

Jancke, Rudolf. Das Wesen der Ironie. Eine Strukturanalyse ihrer Erscheinungsformen, Leipzig, Barth, 1929.

Jankélévitch, V. L'Ironie, Paris, Flammarion, 1964.

Kaiser, W. Praisers of Folly. Erasmus, Rabelais, Shakespeare, Cambridge, Massachusetts, Harvard University Press, 1963.

Longo, A. L'Art du questionnement et les interrogations fictives chez Platon, Milan, Mimesis, 2007.

Mulligan, K. «La varietà e l'unità dell'immaginazione ", Rivista di Estetica, Percezione, 1999, 53-67.

—. "Stupidity, Folly and Cognitive Value », conférence à Kirchberg, 2000.

- _ "Geist (and Gemüt) vs Life - Max Scheler and Robert Musil », Le Ragioni del Conoscere e dell'Agire. Scritti in onore di Rosaria Egidi, R. Calcaterra, dir., Milan, Franco Angeli, 2006, 366-378.

4. Ce texte fut rédigé dans le cadre du NCCR en sciences affectives du Fonds national suisse de la recherche scientifique. Mes remerciements vont à mon traducteur, Guillaume Fréchette, et à Olivier Massin, Ingvar Johansson et Angela Longo pour leurs commentaires. 
—. 2007. "Was sind und was sollen die unechten Gefühlen? ", Hgb. Ursula Amrein, Das Authentische. Zur Konstruktion von Wabrheit in der säkularen Welt, Zürich, Chronos Verlag, à paraître.

Musil, R. Der Mann ohne Eigenschaften. Roman, I, Hgb. A. Frisé, Reinbek bei, Hamburg, Rowohlt, 1983.

—. Der Mann ohne Eigenschaften. Roman. Aus dem Nachlass, II, Hgb. A. Frisé, Reinbek bei Hamburg, Rowohlt, 1983a.

—. Prosa und Stücke. Kleine Prosa, Aphorismen, Autobiographisches, Hgb. A. Frisé, Reinbek bei Hamburg, Rowohlt, 1983b.

Tagebücher. Anmerkungen, Hgb. A. Frisé, Reinbek bei Hamburg, Rowohlt.

Paulhan, J. 1933. La morale de l'ironie, Paris, Alcan, 1983c.

Proust, M. Du côté de chez Swann, A la recherche du temps perdu, Bouquins, Paris, Éditions Robert Laffont, 1987.

Schaerer, R. "Le mécanisme de l'ironie dans ses rapports avec la dialectique ", Revue de métaphysique et de morale, 48, 1941, 181-209.

Scheler, M. "Zum Phänomen des Tragischen ", in Vom Umsturz der Werte, Gesammelte Werke, Bd. 3, Berne, Francke, 149-170, 1955.

Sperber, D. et D. Wilson. Relevance: Communication and Cognition, Oxford, Blackwell, 1986.

White, A. The Language of Imagination, Oxford, Blackwell, 1990. 